Supporting Information

\title{
An Ultrastretchable Reflective Electrode Based on a Liquid Metal Film for Deformable Optoelectronics
}

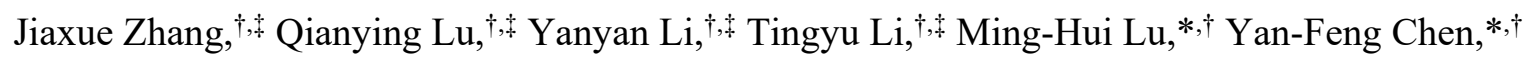
Desheng Kong*,+,

$\uparrow$ College of Engineering and Applied Sciences, National Laboratory of Solid State Microstructure, Collaborative Innovation Center of Advanced Microstructures, and Jiangsu Key Laboratory of Artificial Functional Materials, Nanjing University, Nanjing 210093, China

$\ddagger$ State Key Laboratory of Analytical Chemistry for Life Science, Nanjing University, Nanjing 210093, China

*Correspondence should be addressed to dskong@nju.edu.cn (D.K.), yfchen@nju.edu.cn (Y.C.), and luminghui@nju.edu.cn (M.L.) 


\section{Supporting Figures}

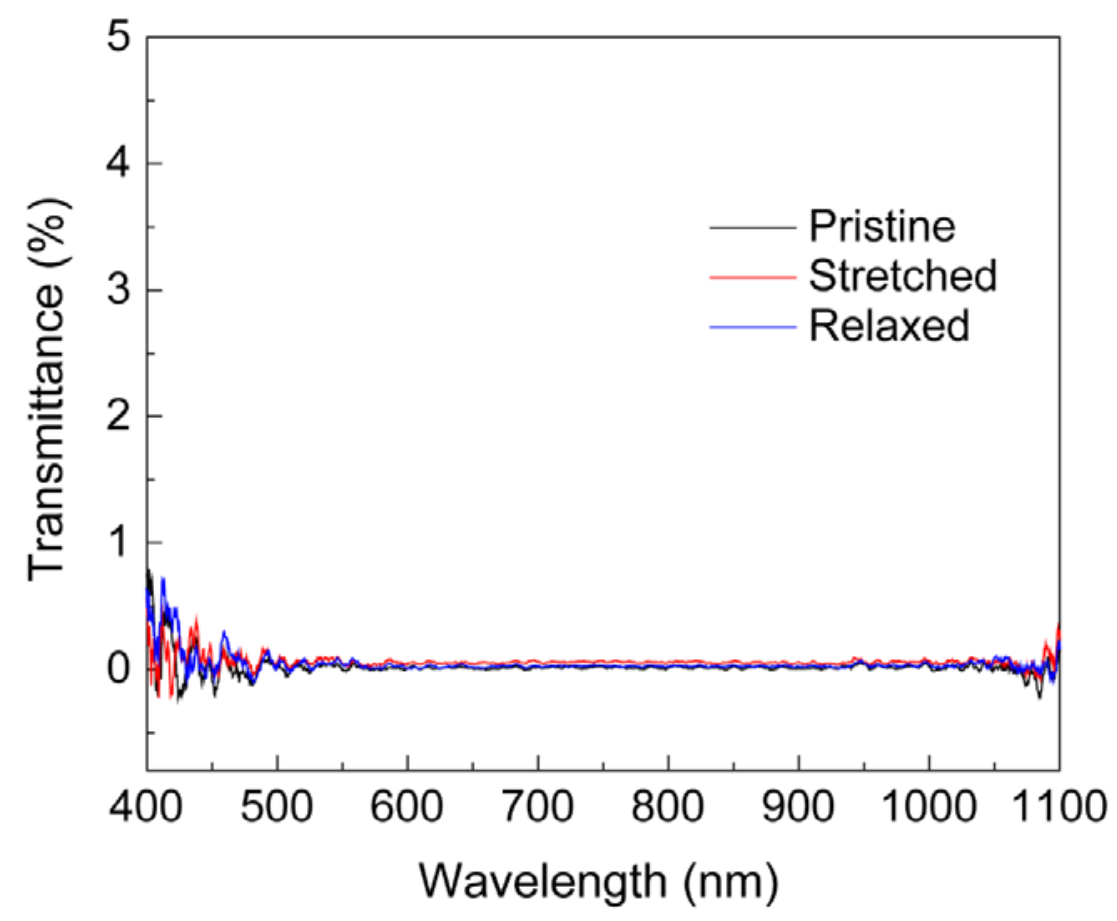

Figure S1. Optical transmittance spectra of liquid metal films at the pristine, stretched (100\% strain), and relaxed states, respectively. The negligible transmittance suggests the absence of dewetting regions as a result of the excellent adhesion of the liquid metal film with SIS substrates.

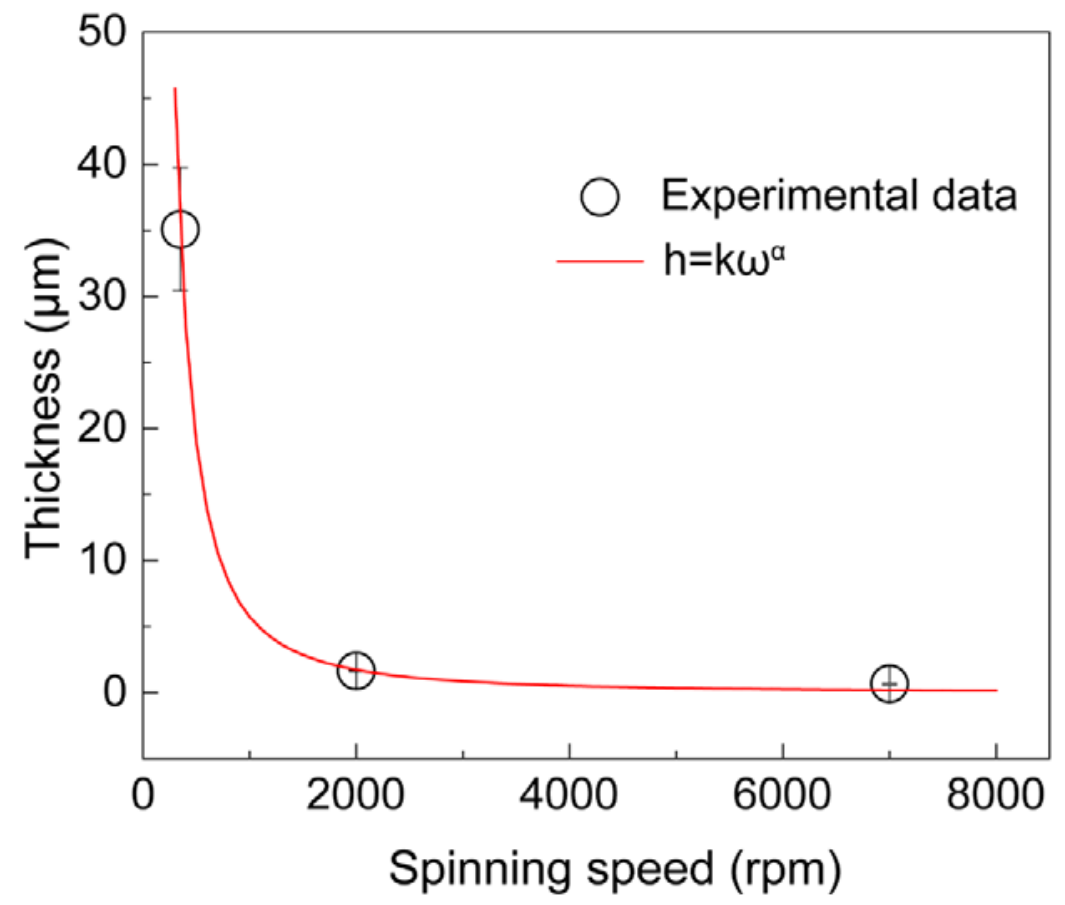

Figure S2. Preparation of liquid metal films with varying thickness by adjusting the spinning speed. The dependence of the thickness $(h)$ on the rotating speed $(\omega)$ is well described by the expression $h=k \omega^{\alpha}$, in which $k$ and $\alpha$ are fitting parameters. ${ }^{1}$ 


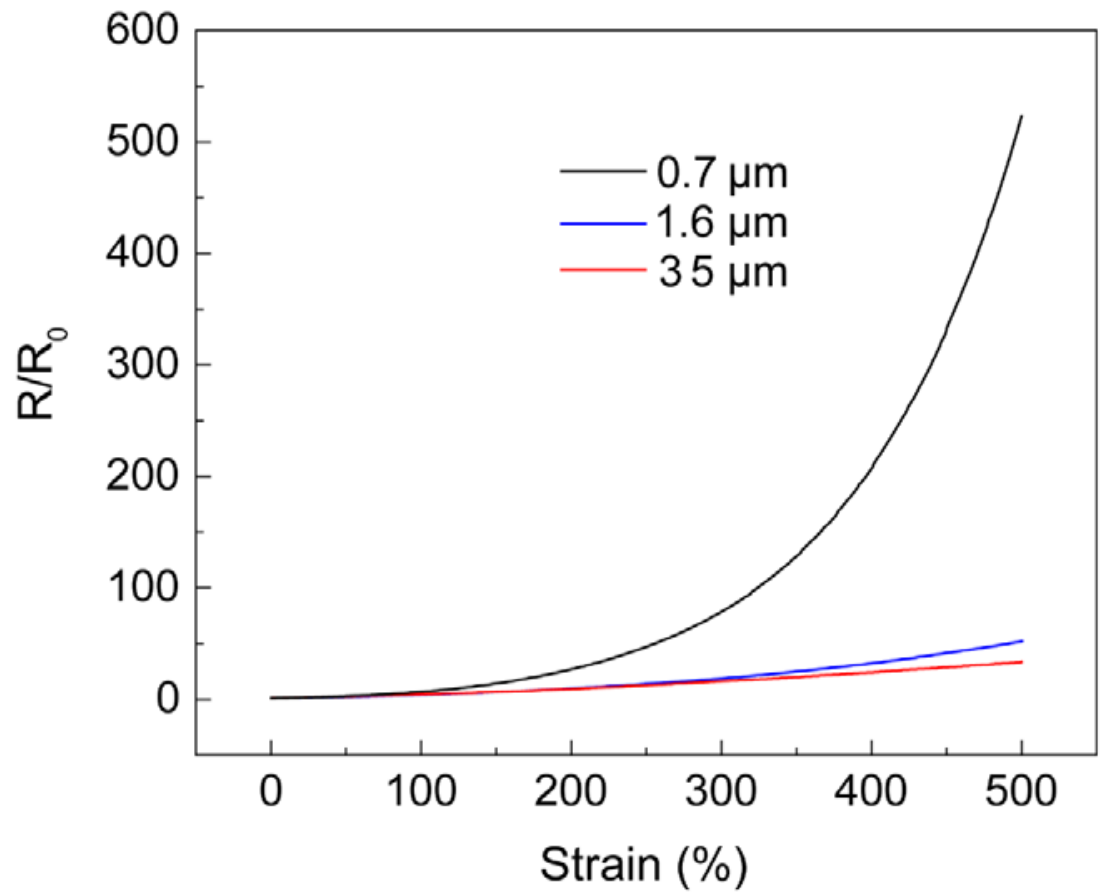

Figure S3. Normalized resistance as a function of tensile strain for liquid metal electrodes with varying thickness. The sheet resistance at relaxed state is $0.23 \Omega \mathrm{sq}^{-1}$ for $0.7 \mu \mathrm{m}$-thick film, $0.15 \Omega$ $\mathrm{sq}^{-1}$ for $1.6 \mu \mathrm{m}$-thick film, and $0.015 \Omega \mathrm{sq}^{-1}$ for $35 \mu \mathrm{m}$-thick film, respectively. The resistance shows larger changes upon stretching likely due to increased impact of surface oxidations on thinner liquid metal film.

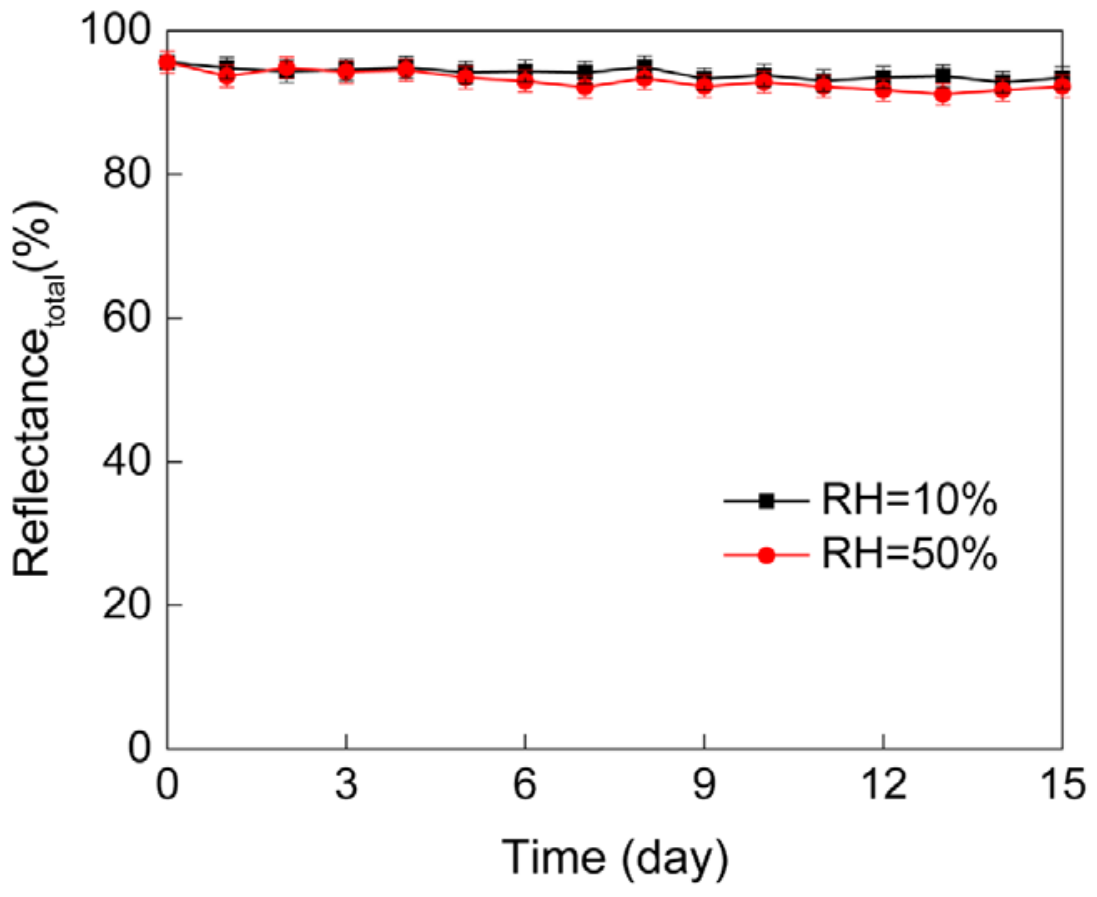

Figure S4. Environmental stability of liquid metal electrodes. Evolution of the total reflectance over 15 day-storage under relative humidity $(\mathrm{RH})$ of $10 \%$ and $50 \%$, respectively. Liquid metal films retain high total reflectance above $90 \%$. 


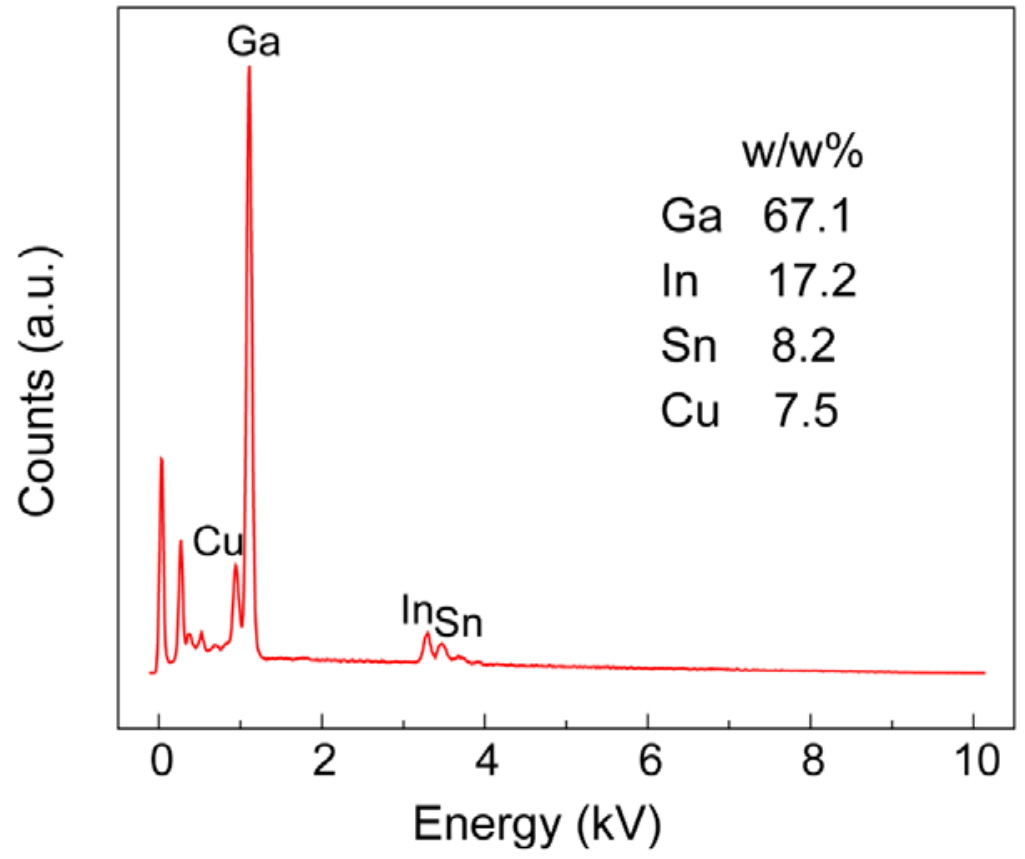

Figure S5. Energy Dispersive X-ray spectroscopy (EDS) spectrum acquired from as-prepared liquid metal film over SIS substrate.
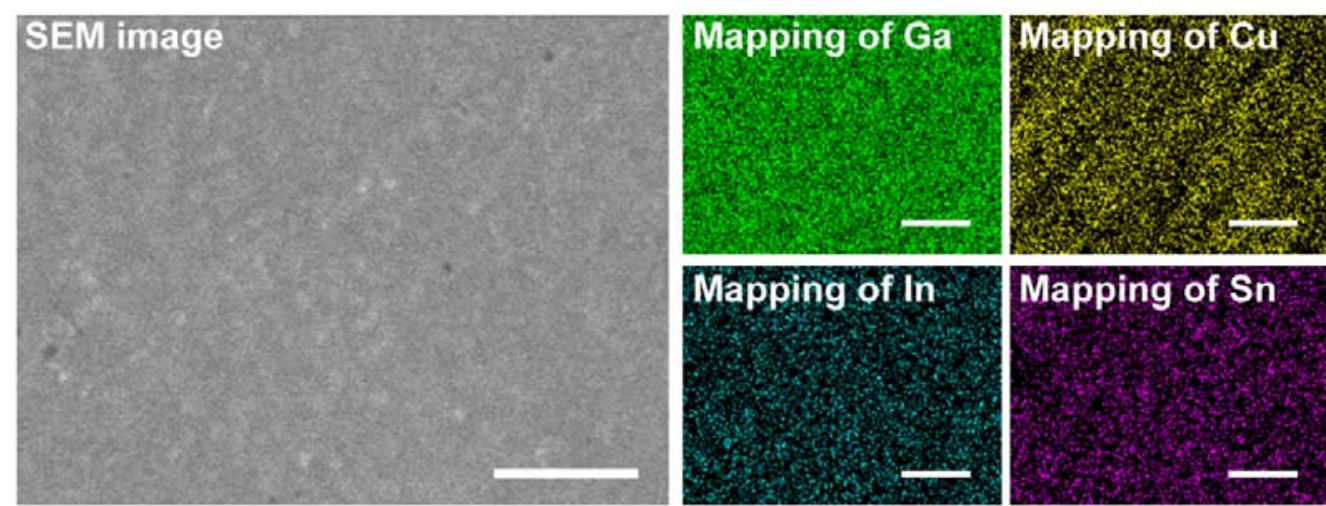

Mapping of Sn

Figure S6. SEM image (left) and corresponding EDS elemental maps (right) acquired from an asprepared liquid metal film. Scale bars: $5 \mu \mathrm{m}$. 

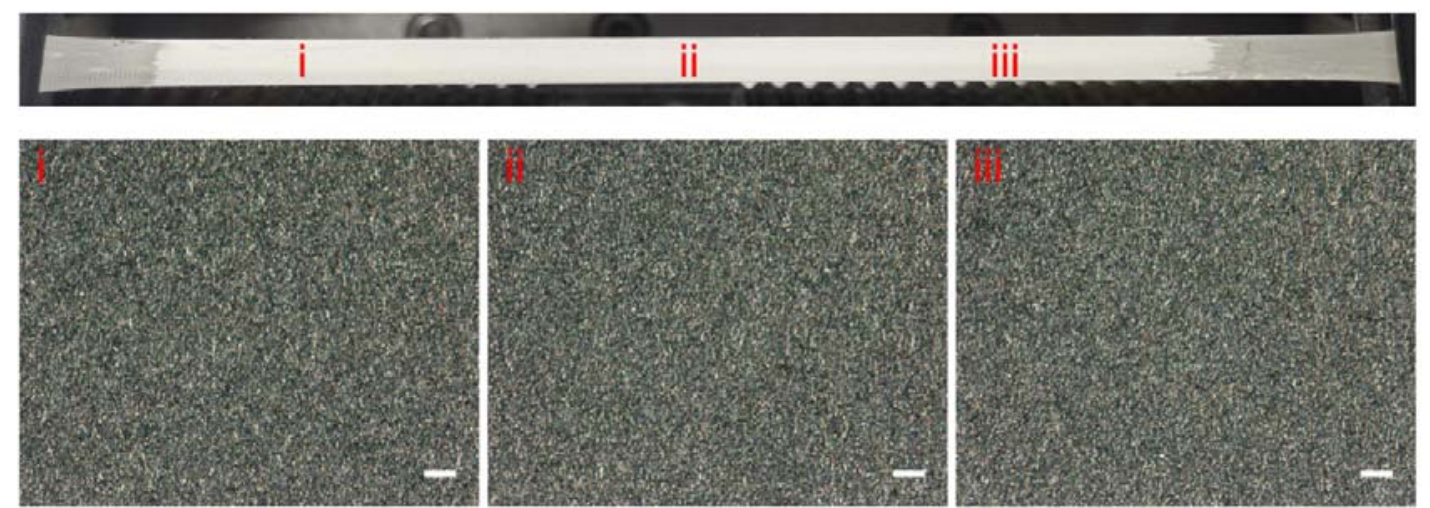

Figure S7. Optical image (top) and corresponding optical microscopy images (bottom) acquired from a $0.7 \mu \mathrm{m}$-thick liquid metal film at 500\% tensile strain. Scale bars: $20 \mu \mathrm{m}$.

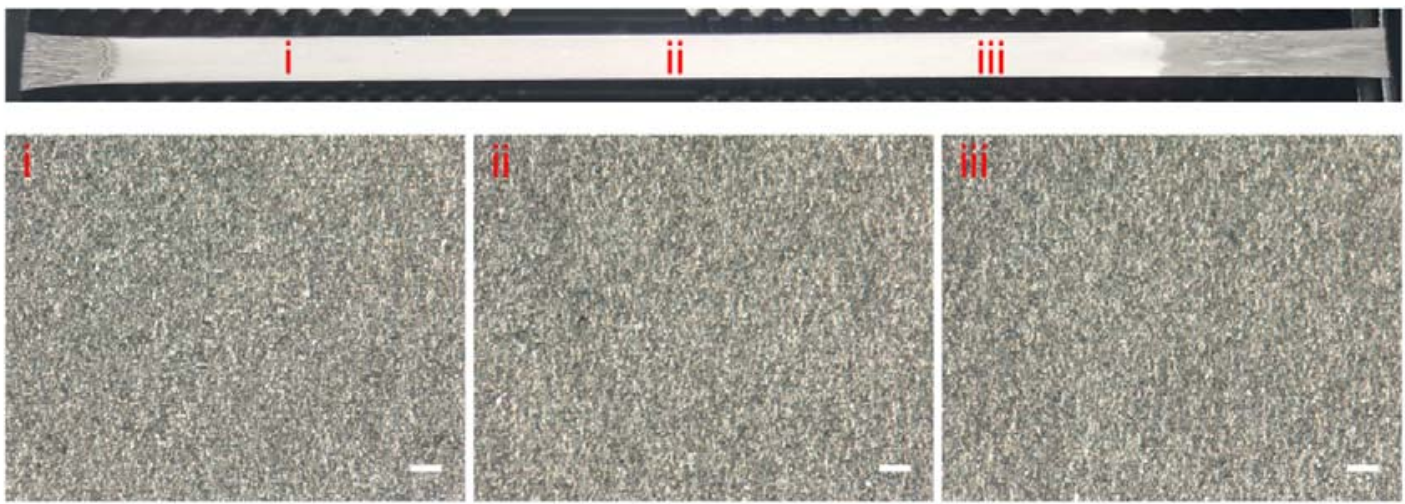

Figure S8. Optical image (top) and corresponding optical microscopy images (bottom) acquired from a $1.6 \mu \mathrm{m}$-thick liquid metal film at 500\% tensile strain. Scale bars: $20 \mu \mathrm{m}$.
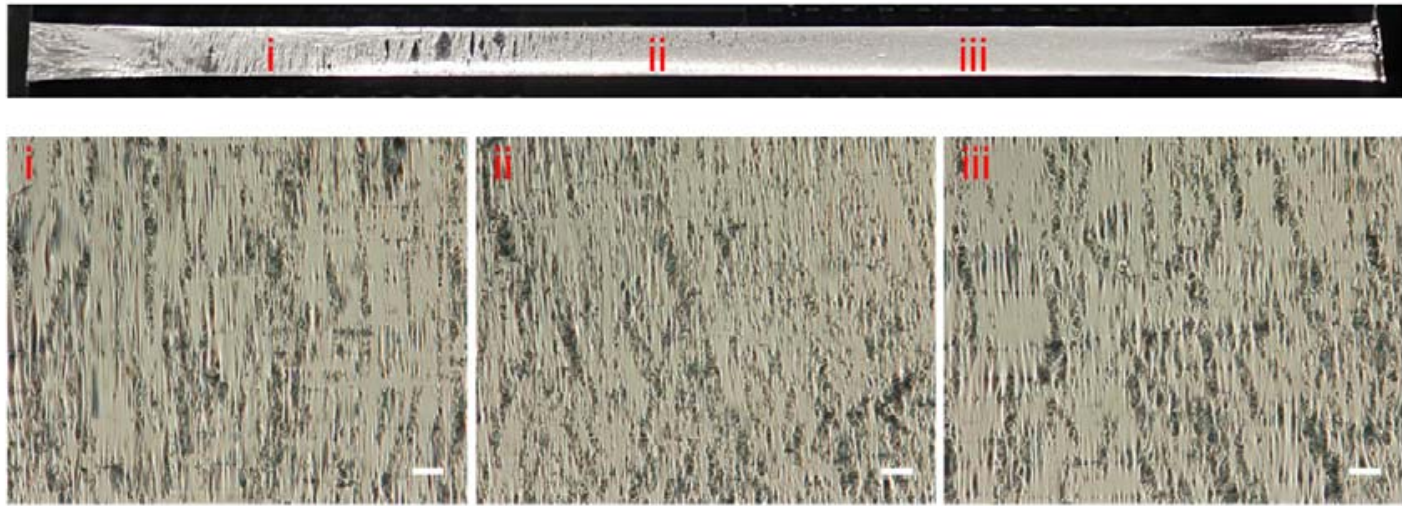

Figure S9. Optical image (top) and corresponding optical microscopy images (bottom) acquired from a $35 \mu \mathrm{m}$-thick liquid metal film at 500\% tensile strain. Scale bars: $20 \mu \mathrm{m}$. The oxide layer is unable to stabilize the surface of thick liquid metal film under large tensile strain due to the tendency for ruptured oxides on sizable liquid metal., ${ }^{2,3}$ 


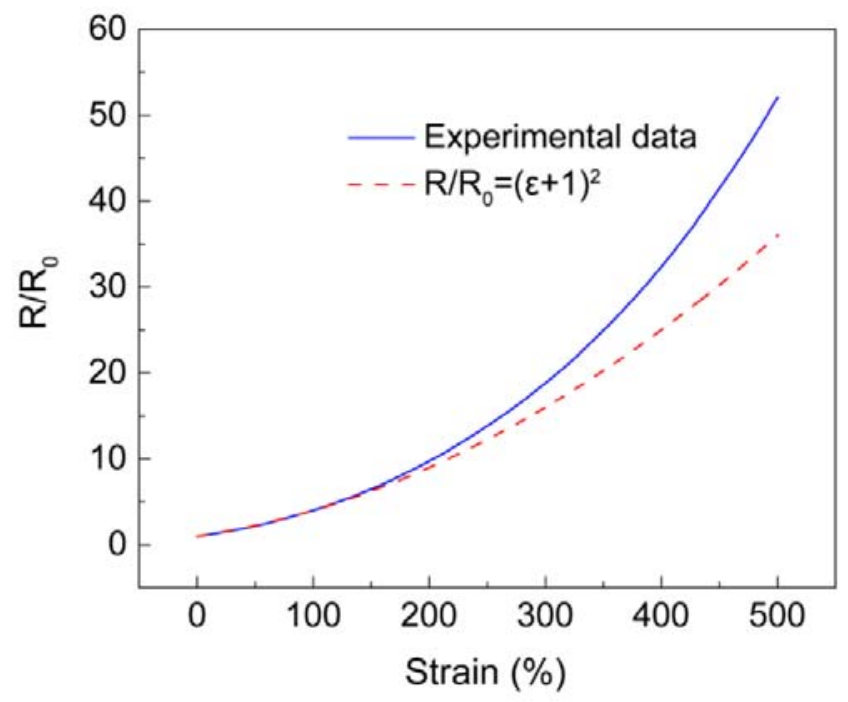

Figure S10. Normalized resistance as a function of tensile strain for as-prepared liquid metal electrode and an ideal liquid-state electrode. Ideal liquid-state conductor exhibits stable conductivity independent of various deformations. The corresponding resistance $(\mathrm{R})$ as a function of uniaxial tensile strain $(\varepsilon)$ is described by the expression $\mathrm{R} / \mathrm{R}_{0}=(\varepsilon+1)^{2} .{ }^{4}$ The additional increase in the resistance of liquid metal electrode than the ideal behavior is ascribed to surface oxidations during tensile deformations.

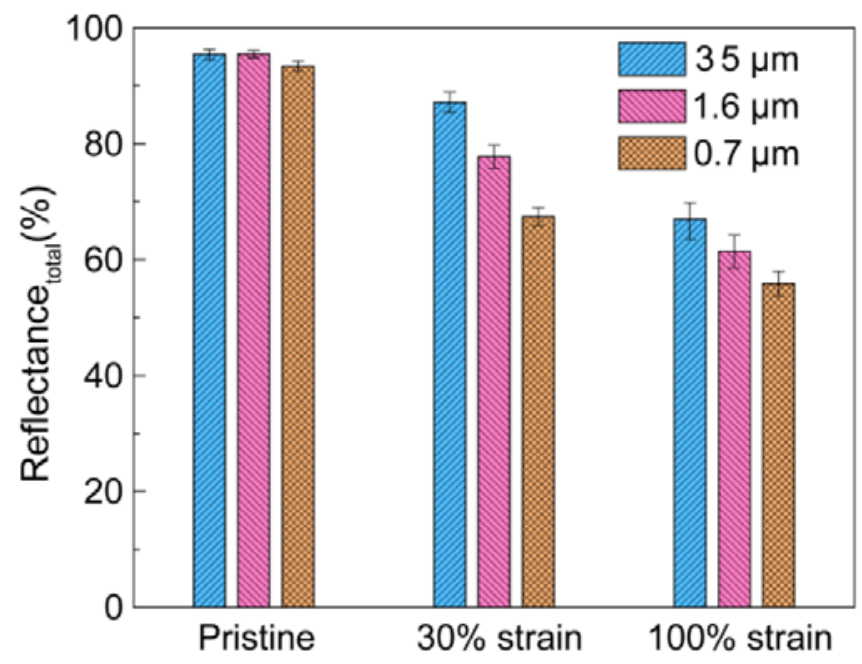

Figure S11. Optical properties of liquid metal electrodes with varying thickness. The total reflectance is $>90 \%$ for all films irrespective of the thickness. After 500 stretch-relaxation cycles, the total reflectance of $0.7 \mu \mathrm{m}$-thick film shows dramatic reductions to $67 \%$ for $30 \%$ strain amplitude and to $56 \%$ for $100 \%$ strain amplitude, respectively. In contrast, the reflective optical properties are largely preserved in $35 \mu \mathrm{m}$-thick liquid metal film after repetitive tensile deformations for 500 cycles. The total reflectance remains at $87 \%$ for $30 \%$ strain amplitude and at $67 \%$ for $100 \%$ strain amplitude, respectively. The drop in reflectance is associated with the increased surface roughness. The oxide layer on sizable liquid metal is easily ruptured by tensile stresses and unable to stabilize the wrinkled textures, ${ }^{2,3}$ consequently resulting in improved retention of the high reflectance in thick liquid metal electrodes. 


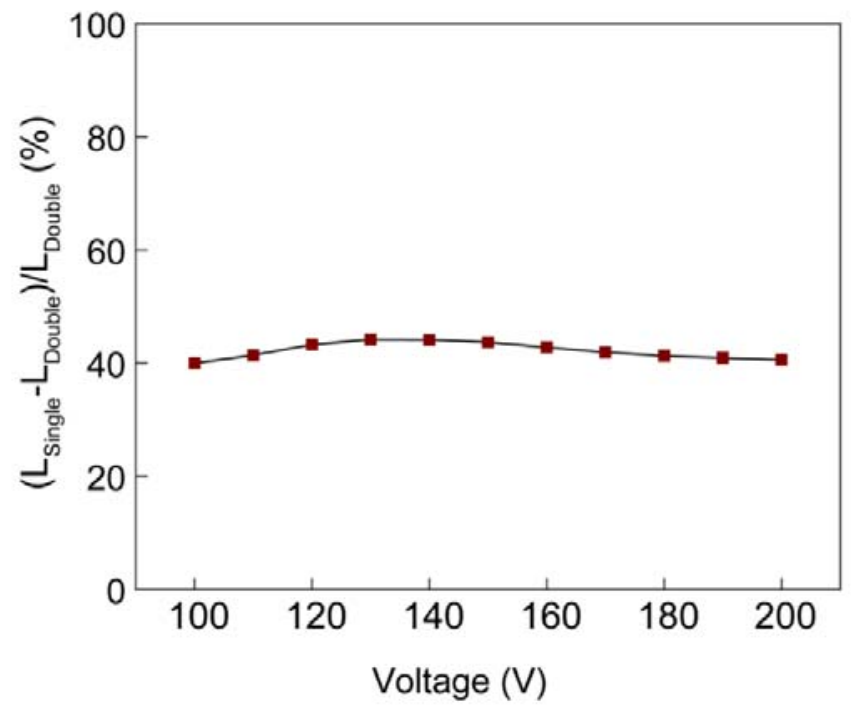

Figure S12. Relative enhancement in the emission intensity of single-side emitting device as compared with double-side emitting device. The light extraction is enhanced by $\sim 40 \%$ under different applied voltages.
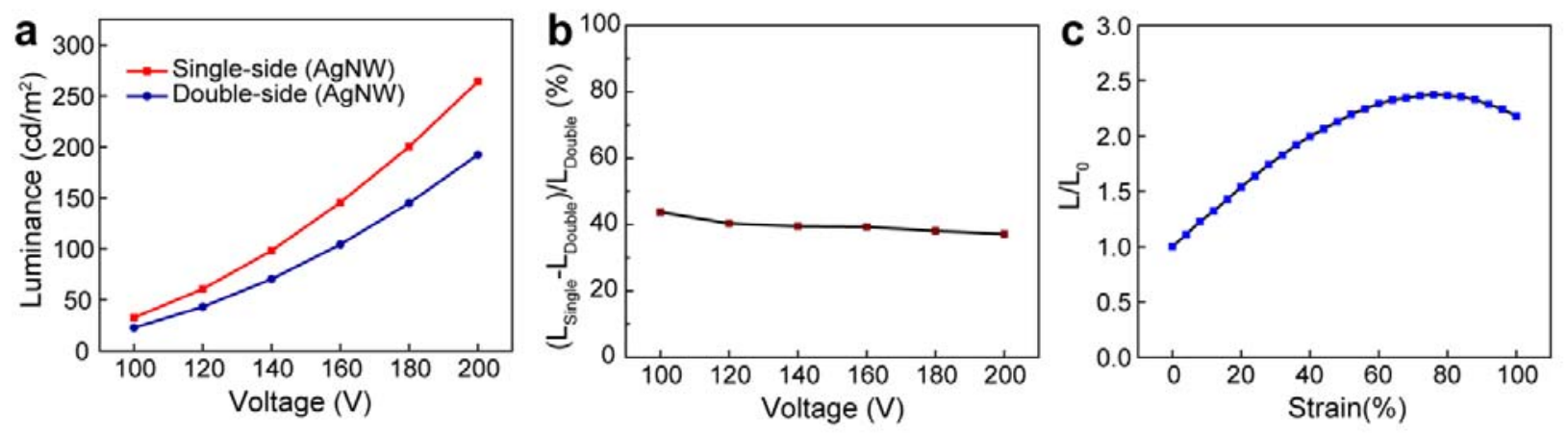

Figure S13. Optoelectronic characteristics of stretchable ACEL devices constructed by using silver nanowire $\left(\mathrm{AgNW}\right.$ ) transparent electrode (Sheet resistance $=1.4 \Omega \mathrm{sq}^{-1}$ and Transmittance at 550nm $=47 \%$ ) and liquid metal reflective electrode. In the device architecture, a $\sim 20 \mu \mathrm{m}$-thick light-emitting layer is employed without any additional dielectric layers due to the inherent low leakage current in AgNW-based devices. (a) Luminance as a function of voltage amplitude of $10 \mathrm{kHz}$ square wave drive voltages. (b) Relative enhancement in the emission intensity of single-side emitting device as compared with double-side emitting device. The value stays at $\sim 40 \%$ irrespective of the applied voltages. As the emission always passes through the top transparent electrode, the relative enhancement is essentially independent of the transmittance value. The transmittance of the transparent electrode inevitably affects the light extraction efficiency and the emission intensity. (c) Normalized luminance as a function of tensile strain for a single-side emitting device. 
a

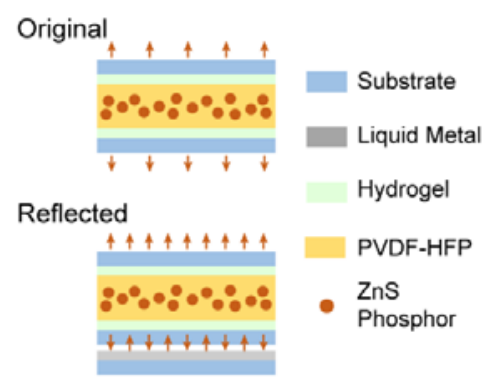

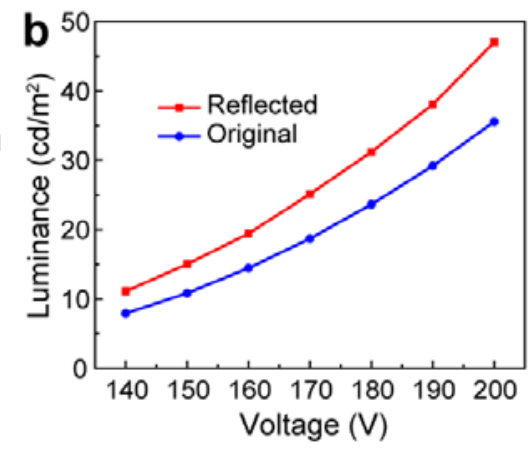

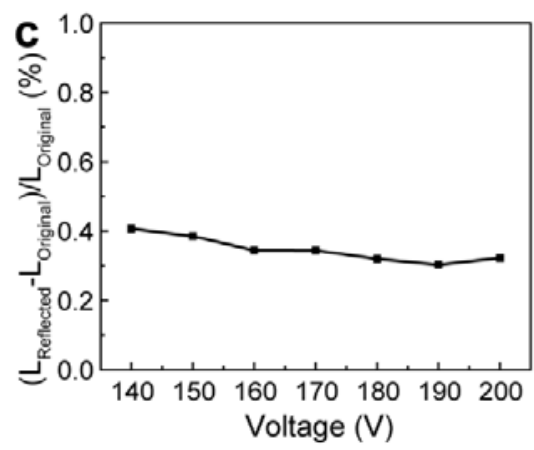

Figure S14. Implementation of liquid metal film as an optical reflector to boost the emission intensity of a double-side emitting ACEL device with two hydrogel transparent electrodes. (a) Schematic architectures of ultrastretchable ACEL devices with double-side (top) and single-side (bottom) emissions. (b) Luminance as a function of voltage amplitude of $10 \mathrm{kHz}$ square wave drive voltages. (c) Relative enhancement in the emission intensity of single-side emitting device as compared with double-side emitting device. The percentage of improvement is slightly less than $40 \%$ and fairly stable at different applied voltages.

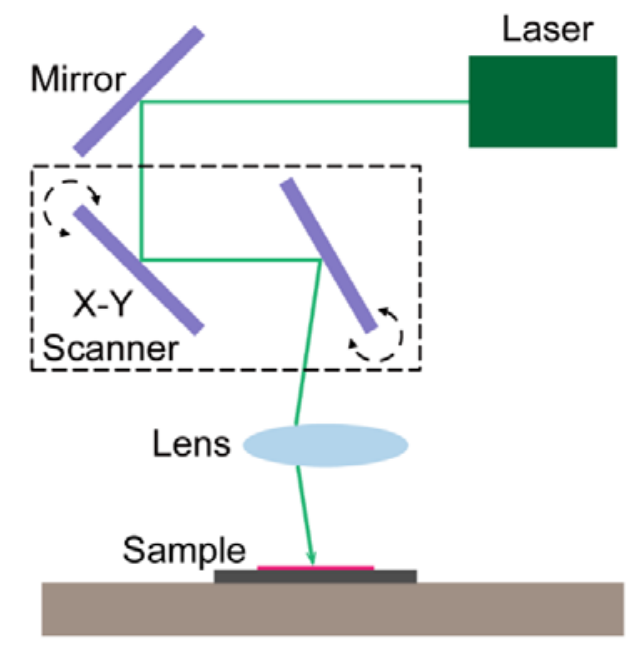

Figure S15. Schematic illustration of the laser marking system for liquid metal selective ablation. 


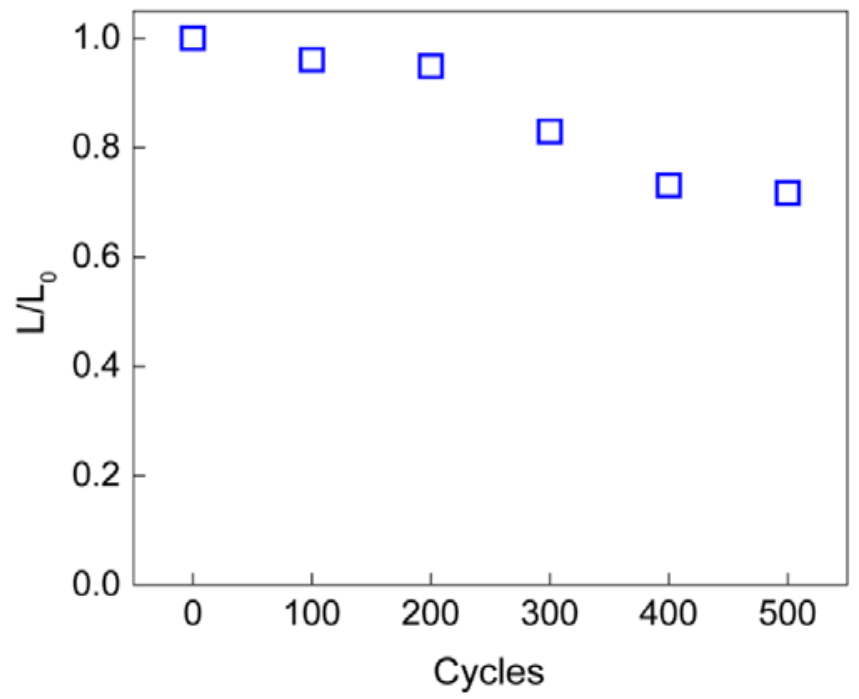

Figure S16. Tensile fatigue test for the single-side emitting device. The normalized luminance is acquired after stretch-relaxation cycles to $100 \%$ strain. In spite of certain decrease in the emission intensity, the device retains the stable operation without any signatures for structural failures.

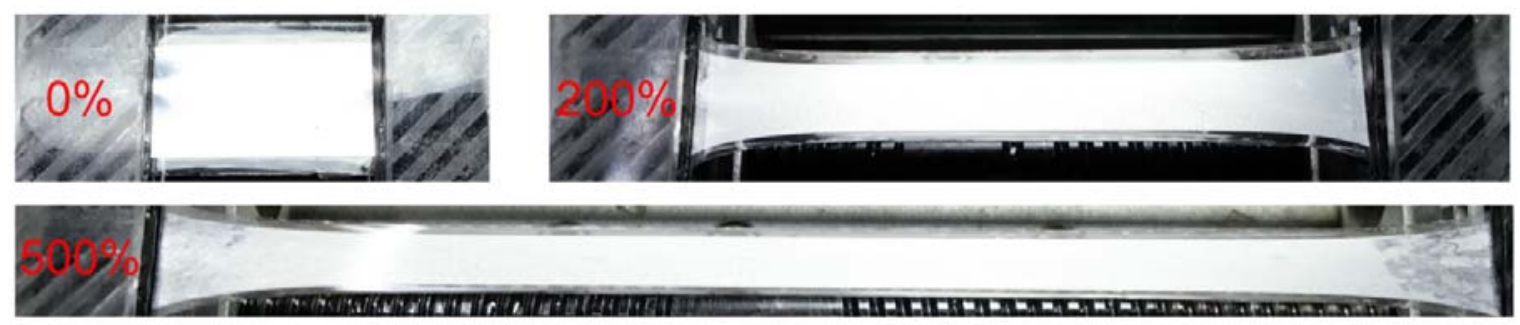

Figure S17. Optical images of liquid metal electrodes encapsulated with a layer of PVDF-HFP elastomer under varying extents of tensile deformations. The liquid metal film exhibits conformal interactions with the elastomeric encapsulation. ${ }^{5}$ There is no identifiable signature for interfacial delamination.

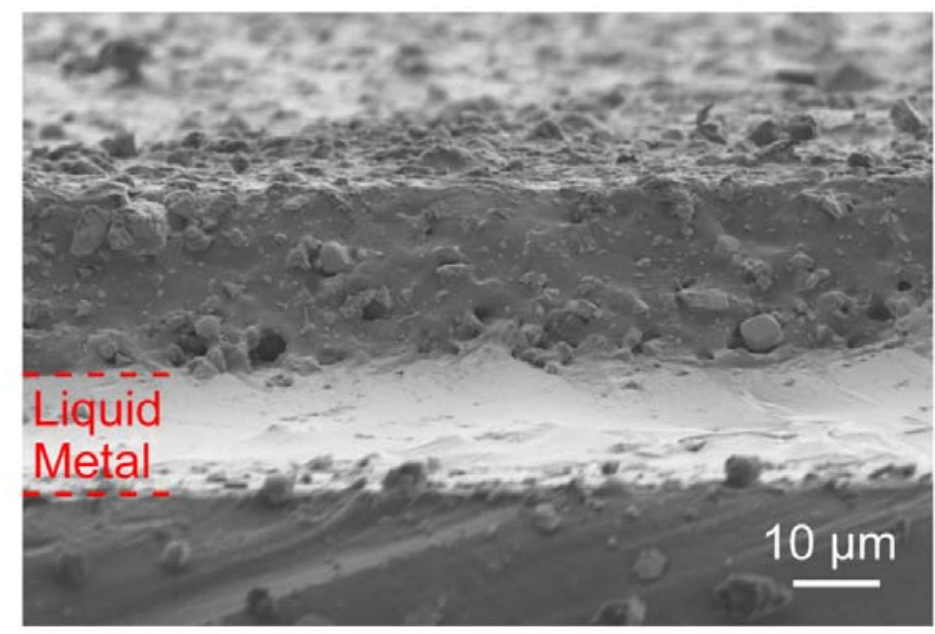

Figure S18. Cross-sectional SEM image of the device stack that reveals intimate contacts of liquid metal film with adjacent functional layers. 

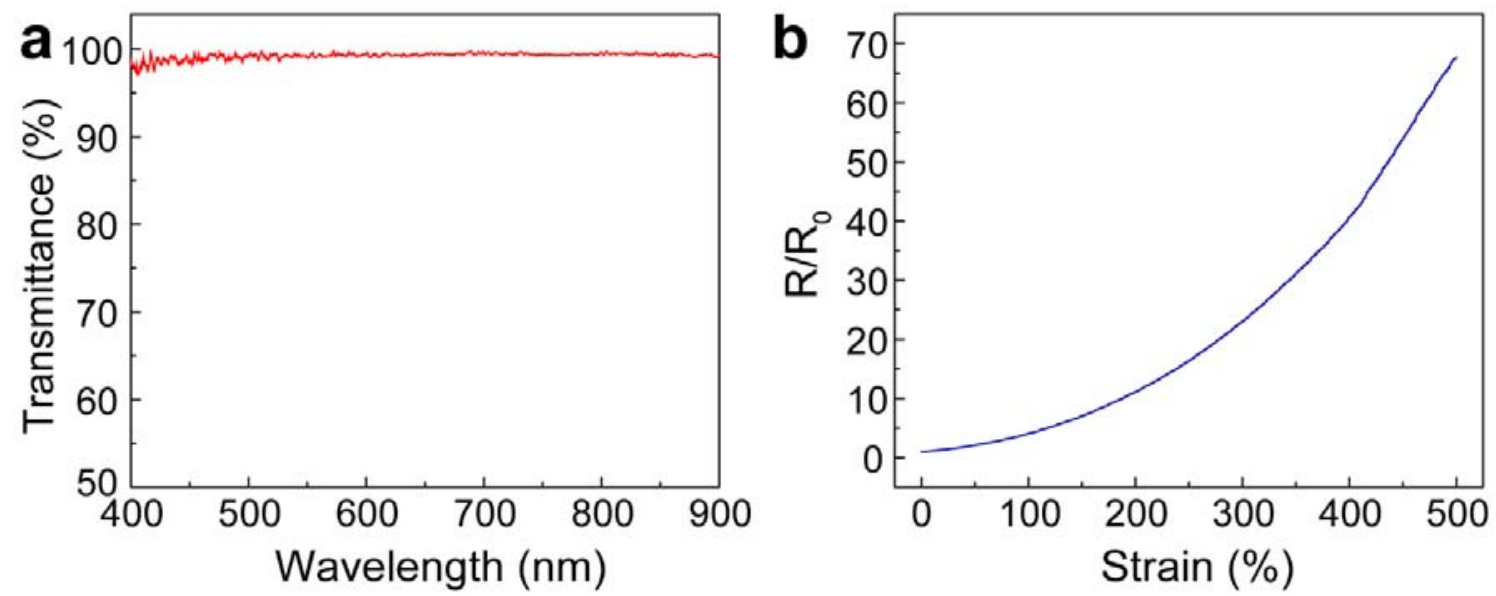

Figure S19. Physical properties of the transparent conductive hydrogel electrode. (a) Transmittance spectrum of an $825 \mu \mathrm{m}$-thick hydrogel electrode in the wavelength range from $400 \mathrm{~nm}$ to $900 \mathrm{~nm}$, which was acquired by a fiber optical spectrometer equipped with an integrating sphere. (b) Normalized resistance as a function of tensile strain. The sheet resistance at relaxed state is measured by a LCR Meter as $183 \Omega \mathrm{sq}^{-1}$.
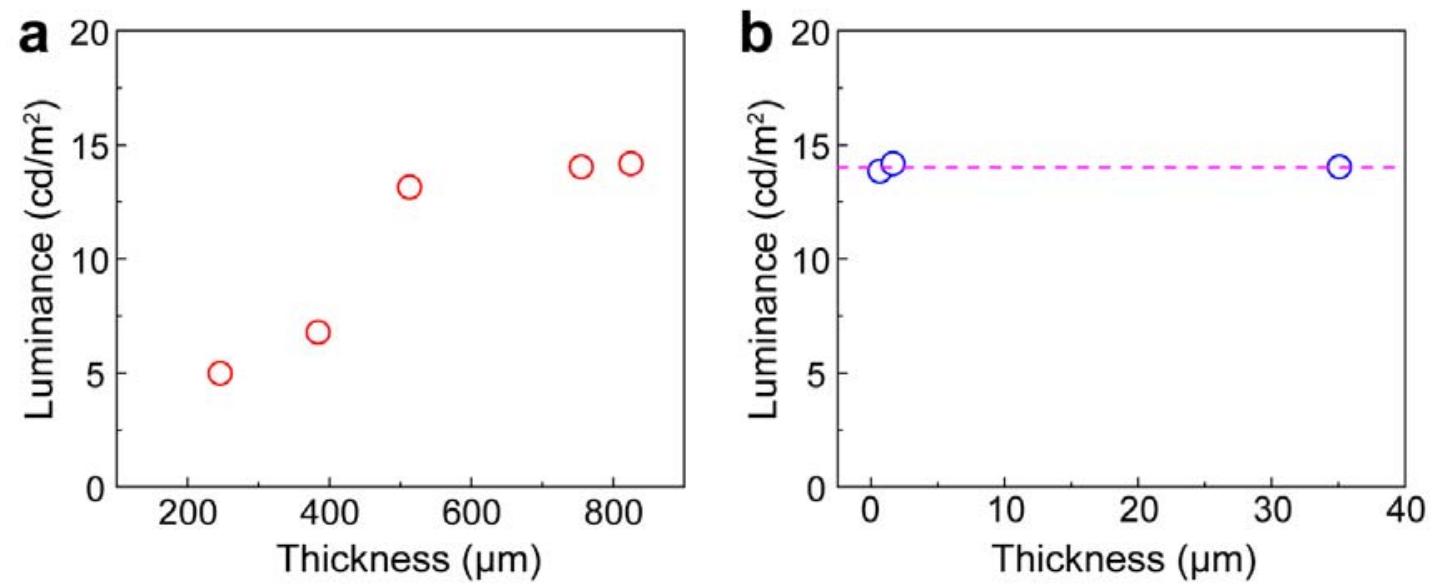

Figure S20. Influence of the thickness of individual electrodes on the emission intensity of singleside emitting devices. (a) Luminance versus the thickness of hydrogel electrodes for light-emitting devices, in which the thickness of liquid metal reflective electrode is fixed at $1.6 \mu \mathrm{m}$. (b) Luminance versus the thickness of liquid metal electrodes for light-emitting devices, in which the thickness of hydrogel is fixed at $825 \mu \mathrm{m}$. All devices are powered by $150 \mathrm{~V}, 10 \mathrm{kHz}$ square wave voltages. Hydrogel electrodes have low ionic conductivity and high sheet resistance $\left(10^{2} \Omega \mathrm{sq}^{-1}\right)$, which give rise to notable voltage drops as practical limiting factors for light emissions. The thickness of hydrogel electrode shows pronounced influence on the emission intensity. Sufficiently thick electrodes (>700 $\mu \mathrm{m})$ are therefore required for the luminance to achieve saturation. In contrast, liquid metal is an electronic conductor with extremely high conductivity $\left(4 \times 10^{4} \mathrm{~S} \mathrm{~cm}^{-1}\right)$. As-prepared liquid metal electrodes show excellent sheet resistance $\left(10^{-2}\right.$ to $\left.10^{-1} \Omega \mathrm{sq}^{-1}\right)$ and negligible influence on the emission intensity. 


\section{Supporting Videos}

Video S1. A $0.7 \mu \mathrm{m}$-thick liquid metal reflective electrode uniaxially deformed from 0 to $500 \%$ strain.

Video S2. A $1.6 \mu \mathrm{m}$-thick liquid metal reflective electrode uniaxially deformed from 0 to $500 \%$ strain.

Video S3. A $35 \mu$ m-thick liquid metal reflective electrode uniaxially deformed from 0 to $500 \%$ strain.

Video S4. A stretchable and single-side emitting ACEL device uniaxially deformed from 0 to $500 \%$ strain.

\section{Supporting References}

1. Koschwanez, J. H.; Carlson, R. H.; Meldrum, D. R. Thin PDMS films using long spin times or tert-butyl alcohol as a solvent. PLoS One 2009, 4, e4572.

2. Tang, L.; Mou, L.; Zhang, W.; Jiang, X. Large-Scale Fabrication of Highly Elastic Conductors on a Broad Range of Surfaces. ACS Appl. Mater. Interfaces 2019, 11, 7138-7147.

3. Li, X.; Li, M.; Zong, L.; Wu, X.; You, J.; Du, P.; Li, C. Liquid Metal Droplets Wrapped with Polysaccharide Microgel as Biocompatible Aqueous Ink for Flexible Conductive Devices. $A d v$. Funct. Mater. 2018, 28, 1804197.

4. Keplinger, C.; Sun, J. Y.; Foo, C. C.; Rothemund, P.; Whitesides, G. M.; Suo, Z. Stretchable, transparent, ionic conductors. Science 2013, 341, 984-987.

5. Doudrick, K.; Liu, S.; Mutunga, E. M.; Klein, K. L.; Damle, V.; Varanasi, K. K.; Rykaczewski, K. Different shades of oxide: from nanoscale wetting mechanisms to contact printing of gallium-based liquid metals. Langmuir 2014, 30, 6867-6877. 